\title{
OCEAN SCIENCES DiRECTORATE OF THE OFFICE OF NAVAL RESEARCH
}

\begin{abstract}
A s of 2 May 1988, the Environmental Sciences Directorate at ONR became the Ocean Sciences Directorate. The list below shows the new organization of divisions and people. The main effect of the reorganization is to separate the physical parts of the old 1122 Ocean Sciences Division into a new ocean and atmospheric physics division (still code 1122), and a new biology/ optics/chemistry division (code 1123). The ocean engineering division now contains an explicit remote sensing division, and the coastal sciences program is now explicitly more interdisciplinary than previously.

New'Accelerated Research Initiatives to start in Fiscal Year 1989:

- Marine Bioluminescence and Upper Ocean Physics and Marine Light- Mixed Layer (MLML)
\end{abstract}

Program Managers: Ann Bucklin, Richard Spinrad. Alan Brandt (Physical Oceanography).

The focus of this interdisciplinary program is to understand and predict space/time variability of the bioluminescent light field in the upper ocean. A central goal is the development of mechanistic models of the interrelated biological, optical, and physical processes. This five-year Accelerated Research Program (ARI) is scheduled for FY8994, and is a follow-on to the successful ARI, "Marine Bioluminescence and Optical Variability in the Water Column" (FY84-89), which has positioned us to make major advances in understanding. Shipboard studies, laboratory experiments, remote sensing, and numerical modeling are all required to achieve the program objectives. The field program will be conducted in the highlatitude North Atlantic, an oceanic region of strong seasonality and physical forcing.

- Ultra-Low-Frequency/Very-Low-Frequency (ULF/VLF) Acoustic Interactions.

Program Managers: Randall Jacobson (Geology/Geophysics); Marshall Orr.

The focus of this program is to understand the space-time variability of the acoustic/seismic ambient noise field in the frequency band from 0.001 to $50 \mathrm{Hertz}$; to identify the noise sources and their noise generating mechanism; and to determine the propagation modes and characteristics for the ambient noise field

- Seafloor Characterization

Program Managers: Joseph Kravitz, Office of Naval Research; Herb Epper, Naval Ocean and Research Development Activity; H. Fleming, Naval Research Laboratory.
The focus of this program is to extend our understanding of oceanographic and geologic processes that control the morphology of the seafloor. The development of the Plate Tectonics syllogism has given us a tool for understanding mega-scale geologic features (e.g.; ridge crests, trenches, seamounts, plateaus) which constrain many oceanographic processes (e.g.; ocean currents, etc.). However, we have a very poor understanding of small (kilometers to centimeters) scale seafloor features which result from these oceanographic and geologic processes.

- Topographic Interactions

Program Managers: Melbourne Briscoe; Ann Bucklin.

Recent observations indicate that dramatic ocean signatures in physical and biological properties are associated with flow over and around abrupt topography; essentially none of these phenomena are adequately predictable with existing models. This interdisciplinary ARI focuses on the flow over Fieberling Guyot. In the near-field there are numerous mixing process (e.g.. internal wave reflection, boundary turbulence, lee waves) that strongly influence the benthic and water-column biological processes. in the far-field there are downstream wakes and biological patchiness. All of these phenomena are strongly influenced by additional motions such as trapped internal waves that circulate around the seamount, and Taylor columns (caused by conservation of potential vorticity) above the seamount; the effects of many of the phenomena can be sensed from space or by optical or acoustic in situ instruments. Laboratory and computer modeling is being used to help define the scientific issues and appropriate field experiments and will be essential in the synthesis of the physical and biological data sets. The goal of the seamount study is to be able to predict oceanic phenomena near strong topography. A secondary focus of the overall program is topographic stress (generalized form drag); i.e., how does one include sub-grid scale topography in numerical models of the ocean circulation in a physically and mathematically consistent manner?

Highlights of the Ocean Acoustics Program (ONR Code $11250 A$, Marshall Orr, Acting Program Manager).

The acoustics program funds scientists in the academic sector to understand the physics of acoustic and elastic wavefield propagation and scatter in the ocean environment. The primary 
purpose of the program is to develop an ability to predict in a quantitative manner the acoustic wavefield, which may be influenced by the entire ocean environment. The ocean environment includes the ocean volume, the ocean boundaries (air/sea, bottom), and the surrounding media (atmosphere and subbottom). Wavefield propagation and scatter in the ocean environment is controlled by spatial and temporal changes in the medium's refractive properties.

The study of ocean acoustics requires an understanding of the deterministic and statistical properties (in both a spatial and temporal sense) of the propagating media. An ocean acoustician must understand the physics of acoustic and elastic wavefield propagation, fluid dynamics on a variety of spatial scales, geology and geophysics (i.e., processes which form the ocean bottom and subbottom and the heterogeneity of the bottom and subbottom elastic and anelastic properties) and marine biology (size and distribution of animals and plants that scatter sound).

Consequently, the proper characterization of the propagating and scattering media requires ocean acousticians to work on an interdisciplinary basis with physical oceanographers, geologists and geophysicists, biological oceanographers, remote sensing experts and meteorologists.

The frequency band of interest to the ocean acoustics program ranges from $10^{-3}$ to $10^{7} \mathrm{hz}$. The wavelength of the signals ranges from $1500 \mathrm{~km}$ to $1.5 \mathrm{~mm}$. Spatial variability of the refractive properties of the propagating medium occurs on a similarly wide range of scales. The three dimensional and temporal changes of the acoustic wavefield propagation and scattering are of interest to the program. A quantitative understanding of these phenomena challenges existing experimental techniques, existing theory, and the largest serial super-computers.
Unlike other physical sciences which are taught on a disciplinary basis at the graduate and undergraduate level, ocean acoustics is learned by selfstudy or at universities which have one or two scientists whose research interests include ocean acoustics. Many ocean acousticians, who typically received primary training as physicists, learned ocean acoustics either during World War II or from people who were immediately involved in that effort. Consequently, the population of ocean acousticians within the academic sector is advancing in age and decreasing in number. The Navy is also concerned that, with the exception of the MIT-WHOI Joint Program, there are few educational centers that train young ocean acousticians and researchers; furthermore, the majority of those trained are theoretically oriented, with fewer gaining needed experience at sea and with instruments.

ONR's acoustic program, which is the only domestic source of ocean acoustic basic research funds, will be attempting to address the educational needs of the ocean acoustics discipline. This will be done by funding research at a limited number of institutions that have the critical mass of experimental, theoretical, and numerical ocean acousticians who can interact with ocean -going and theoretical physical oceanographers, geologists, geophysicists, and biological oceanographers.

In addition, the program will, when possible. respond to proposals submitted by young academic scientists attempting to enter the ocean acoustics discipline, especially if these young scientists attempt to develop collaborative and interdisciplinary ocean acoustics research with established members (mentors) of the ocean acoustics research community.

Contributed by Melbourne Briscoe and Leonard Johnson.

\section{OCEAN SCIENCES DIRECTORATE}

Director: Dr. Eric Hartwig, Code 112; Deputy Director: Cdr. Thomas Nelson, Code 112D

Ocean Engineering Division

Director - vacant (Dr. Steven Ramberg. acting), Code 1121; Program Manager, Ocean Technology: Dr. Steven Ramberg, Code 11210T; Program Manager, Research Facilities: Mr. Keith Kaulum. Code 1121RF; Program Manager, Coastal Sciences: Dr. Alan Brandt, Code $1121 \mathrm{CS} ;$ Scientific Officer: Dr. Raymond Fitzgerald; Program

Manager, Remote Sensing: Dr. Frank Herr, Code 1121RS: Scientific Officers: Mr. Hans Dolezalek, Mr. Charles Luther

Ocean and Atmospheric Physics Division

Director - Dr. Alan Weinstein, Code 1122: Program Manager, Meso/Large Scale Physical Oceanography: Dr. David Evans, Code 1122ML; Scientific Officers: Dr. Thomas Kinder, Dr. Melbourne Briscoe (IPA); Program Manager, Small Scale Physical Oceanography: Dr. Alan Brandt (acting), Code 1122SS; Scientific Officers: Mr. William Cross, Dr. Norden Huang (Detail); Program Manager, Marine Meteorology: Dr. Robert Abbey, Code $1122 \mathrm{MM}$

Biology/Optics/Chemistry Division

Director - vacant (Dr. Richard Spinrad, acting), Code 1123; Program Manager, Oceanic Biology: Dr. Ann Bucklin, Code 1123B: Scientific Officers: Dr. Bernard Zahuranec, Dr. Randall Alberte (IPA); Program Manager, Ocean Optics: Dr. Richard Spinrad, Code 1123OP, Program Manager, Oceanic Chemistry: Dr. Edward Green, Code 1123C Geo-Acoustics/Arctic Division

Director - Dr. Leonard Johnson, Code 1125; Program Manager. Ocean Acoustics: Dr. Marshall Orr, Code 1125OA; Scientific Officer: Mr. Marvin Blizard; Program Manager, Geology \& Geophysics: Dr. Joseph Kravitz, Code 1125GG; Scientific Officers: Mr. Jack Heacock, Dr. Randall Jacobson (IPA); Program Manager, Arctic Sciences: Dr. Thomas Curtin, Code 1125AR'; Scientific Officers: Mr. Robert Obrochta, Mr. Dean Horn (IPA) 